\title{
Traces of uniform families of sets
}

\author{
Balázs Patkós* \\ Submitted: Aug 24, 2008; Accepted: Feb 23, 2009; Published: Mar 4, 2009 \\ AMS Mathematics Subject Classification: 05D05
}

\begin{abstract}
The trace of a set $F$ on a another set $X$ is $\left.F\right|_{X}=F \cap X$ and the trace of a family $\mathcal{F}$ of sets on $X$ is $\mathcal{F}_{X}=\left\{\left.F\right|_{X}: F \in \mathcal{F}\right\}$. In this note we prove that if a $k$-uniform family $\mathcal{F} \subset\left(\begin{array}{c}{[n]} \\ k\end{array}\right)$ has the property that for any $k$-subset $X$ the trace $\left.\mathcal{F}\right|_{X}$ does not contain a maximal chain (a family $C_{0} \subset C_{1} \subset \ldots \subset C_{k}$ with $\left|C_{i}\right|=i$ ), then $|\mathcal{F}| \leq\left(\begin{array}{c}n-1 \\ k-1\end{array}\right)$. This bound is sharp as shown by $\left\{F \in\left(\begin{array}{c}{[n]} \\ k\end{array}\right), 1 \in F\right\}$. Our proof gives also the stability of the extremal family.
\end{abstract}

\section{Introduction}

Let $[n]$ denote the set of the first $n$ positive integers $\{1,2, \ldots, n\}$. Given a set $X$ we write $2^{X}$ for its power set and $\left(\begin{array}{c}X \\ l\end{array}\right)$ for the set of all of its $l$-element subsets (l-subsets for short). Given a family $\mathcal{F} \subseteq 2^{X}$ of sets and an element $x \in X$ we write $\mathcal{F}_{x}$ for the subfamily of all the sets in $\mathcal{F}$ that contain $x$ and $\mathcal{F}_{\bar{x}}$ for the family $\left\{F \backslash\{x\}: F \in \mathcal{F}_{x}\right\}$. The degree of $x$ is the size of $\mathcal{F}_{x}$.

The trace of a set $F$ on another set $X$ is $F \cap X$ and is denoted by $\left.F\right|_{X}$. The trace of a family $\mathcal{F}$ of sets is just the family of traces, i.e. $\left.\mathcal{F}\right|_{X}=\left\{\left.F\right|_{X}: F \in \mathcal{F}\right\}$. The following fundamental result concerning traces of families was proved in the early 1970s independently by Sauer [11], Shelah [12] and Vapnik and Chervonenkis [13].

Theorem 1.1. If $\mathcal{F} \subseteq 2^{[n]}$ is a family with more than $\sum_{i=0}^{k-1}$ sets, then there exists a $k$-subset $X$ of $[n]$ such that $\left.\mathcal{F}\right|_{X}=2^{X}$.

The above theorem is sharp as shown by the families $\{F \subseteq[n]:|F|<k\}$ and $\{F \subseteq[n]:|F|>n-k\}$, but no characterization is known for the extremal families. Füredi and Quinn [7] constructed extremal families $\mathcal{F}_{l}$ of size $\sum_{i=0}^{k-1}$ for all $l$ with $0<l<k$ such that for any $k$-subset $X$ of $[n]$ we have $\left.\left(\begin{array}{c}X \\ l\end{array}\right) \nsubseteq \mathcal{F}\right|_{X}$.

Frankl and Pach [5] considered the $k$-uniform case of the problem. They proved the following upper bound.

*Department of Computer Science, Eötvös Loránd University, Budapest, 1117 Pázmány Péter sétány 1/C, Hungary. Email: patkosb@cs.elte.hu. Supported by OTKA NK 67867. 
Theorem 1.2. If $\mathcal{F} \subseteq\left(\begin{array}{c}{[n]} \\ k\end{array}\right)$ and $|\mathcal{F}|>\left(\begin{array}{c}n \\ k-1\end{array}\right)$, then there is a k-subset $X$ of $[n]$ such that $\left.\mathcal{F}\right|_{X}=2^{X}$.

Frankl and Pach conjectured $\left\{F \in\left(\begin{array}{c}{[n]} \\ k\end{array}\right): 1 \in F\right\}$ to be an extremal family of size $\left(\begin{array}{l}n-1 \\ k-1\end{array}\right)$, but Ahlswede and Khachatrian [1] disproved their conjecture by giving a counterexample of size $\left(\begin{array}{l}n-1 \\ k-1\end{array}\right)+\left(\begin{array}{l}n-4 \\ k-3\end{array}\right)$. Later Mubayi and Zhao [9] gave exponentially many pairwise nonisomorphic families of that size and improved the upper bound of Frankl and Pach, but the problem is still open.

Several papers [2], [3], [10] dealt with "Turán-type" problems of traces, i.e. given one or more families $\mathcal{H}_{1}, \mathcal{H}_{2}, \ldots, \mathcal{H}_{s} \subseteq 2^{[h]}$ what is the maximum size of a family $\mathcal{F} \subseteq 2^{[n]}$ such that for any $h$-subset $X$ of $[n]$ and $1 \leq i \leq s$ the trace $\left.\mathcal{F}\right|_{X}$ does not contain $\mathcal{H}_{i}$. With this formulation in Theorems 1.1 and 1.2 the excluded family is $2^{[k]}$.

In [10] it is proved (among others) that if we change $2^{[k]}$ to the maximal chain $\mathcal{C}_{k}=$ $\{\emptyset,[1],[2], \ldots,[k]\}$ in Theorem 1.1, then the only extremal families are $\{F \subseteq[n]:|F|<k\}$ and $\{F \subseteq[n]:|F|>n-k\}$. In this note we consider the corresponding $k$-uniform problem and prove that the conjecture of Frankl and Pach becomes true in this scenario if again we change $2^{[k]}$ to $\mathcal{C}_{k}$. Furthermore we prove the stability of the extremal family $\left\{F \in\left(\begin{array}{c}{[n]} \\ k\end{array}\right): 1 \in F\right\}$.

Theorem 1.3. For every integer $2 \leq k$ and real $1 / 2<c<1$ there exists an $N_{0}(k, c)$ such that for any $n \geq N_{0}(k, c)$ if $\mathcal{F} \subseteq\left(\begin{array}{c}{[n]} \\ k\end{array}\right)$ has size larger than $c\left(\begin{array}{c}n-1 \\ k-1\end{array}\right)$ and there is no subset $X$ of $[n]$ with $|X|=k$ such that $\left.\mathcal{C}_{k} \subseteq \mathcal{F}\right|_{X}$, then there exists an $x \in[n]$ such that $x \in F$ for all $F \in \mathcal{F}$.

Clearly Theorem 1.3 is a generalization of the well-known Erdös-Ko-Rado theorem [4], therefore it is not surprising that our proof will use the following stability theorem of Hilton and Milner [8].

Theorem 1.4. If $2 k+1 \leq n$ and $\mathcal{F} \subseteq\left(\begin{array}{c}{[n]} \\ k\end{array}\right)$ is an intersecting family such that $\bigcap_{F \in \mathcal{F}} F=\emptyset$, then $|\mathcal{F}| \leq\left(\begin{array}{l}n-1 \\ k-1\end{array}\right)-\left(\begin{array}{c}n-k-1 \\ k-1\end{array}\right)+1$.

\section{Proof of Theorem 1.3}

First we prove a lemma stating that if we want to have an "almost" maximal chain $\mathcal{C}_{k}^{-}=\{[1],[2], \ldots,[k]\}$ as trace, then much smaller families suffice.

Lemma 2.1. For every integer $2 \leq k$ and real $1 / 2<c^{\prime}<1$ there exists an $N_{0}^{\prime}\left(k, c^{\prime}\right)$ such that for any $n \geq N_{0}^{\prime}\left(k, c^{\prime}\right)$ if $\mathcal{F} \subseteq\left(\begin{array}{c}{[n]} \\ k\end{array}\right)$ has size larger than $c^{\prime}\left(\begin{array}{c}n-1 \\ k-1\end{array}\right)$ then there exists a set $X \subset[n]$ with $|X|=k$ such that $\left.\mathcal{C}_{k}^{-} \subseteq \mathcal{F}\right|_{X}$.

Proof of Lemma: We proceed by induction on $k$. For $k=2$, if there exists an intersecting pair of 2-sets $F_{1}, F_{2} \in \mathcal{F}$, then $\emptyset \neq\left. F_{1}\right|_{F_{2}} \subset F_{2}$ is a $C_{2}^{-}$. Therefore $\mathcal{F}$ is a pairwise disjoint family and thus $|\mathcal{F}| \leq n / 2<c^{\prime}(n-1)$ for any $1 / 2<c^{\prime}$ if $n$ is large enough.

Now suppose the lemma is proved for $k-1$ and any real between $1 / 2$ and 1 . For a real $c^{\prime}$ fix an $M>N^{\prime}\left(k-1, \frac{c^{\prime}+1 / 2}{2}\right)$ such that the following inequalities hold for all $n \geq M$ 


$$
\begin{gathered}
\frac{c^{\prime}-1 / 2}{2}\left(\begin{array}{l}
n-2 \\
k-2
\end{array}\right)>\left(\begin{array}{l}
n-2 \\
k-2
\end{array}\right)-\left(\begin{array}{c}
n-k-2 \\
k-2
\end{array}\right), \\
c^{\prime}\left(\left(\begin{array}{l}
n-2 \\
k-2
\end{array}\right)+\left(\begin{array}{l}
n-3 \\
k-2
\end{array}\right)\right)>\left(\begin{array}{l}
n-2 \\
k-2
\end{array}\right) .
\end{gathered}
$$

The existence of such $M$ for (1) follows from the fact that if we consider the two sides of (1) as polynomials of $n$, then the degree of the LHS is one larger than the degree of the RHS and for (2) from $c^{\prime}>1 / 2$ and from $\lim _{n \rightarrow \infty}\left(\begin{array}{l}n-2 \\ k-2\end{array}\right) /\left(\begin{array}{l}n-3 \\ k-2\end{array}\right)=1$.

Let $N^{\prime}\left(k, c^{\prime}\right)=M+1+2\left(\begin{array}{c}M+1 \\ k-1\end{array}\right), n \geq N^{\prime}\left(k, c^{\prime}\right)$ and $\mathcal{F} \subseteq\left(\begin{array}{c}{[n]} \\ k\end{array}\right)$ a family with $|\mathcal{F}| \geq c^{\prime}\left(\begin{array}{c}n-1 \\ k-1\end{array}\right)$. Let $x_{1} \in[n]$ be an element with maximum degree which is at least the average degree $c^{\prime}\left(\begin{array}{l}n-1 \\ k-1\end{array}\right) \frac{k}{n} \geq c^{\prime}\left(\begin{array}{l}n-2 \\ k-2\end{array}\right)$ and consider $\mathcal{F}_{\bar{x}_{1}}$. By the inductive hypothesis there exists a $(k-1)$ subset $X \subset[n] \backslash\left\{x_{1}\right\}$ such that $\left.\mathcal{F}_{\bar{x}_{1}}\right|_{X}$ contains $\mathcal{C}_{k-1}^{-}$. Just by removing these sets one after the other and repeatedly using the inductive hypothesis we get that $\mathcal{G}=\left\{X \in \mathcal{F}_{\bar{x}_{1}}\right.$ : $\left.\left.\mathcal{C}_{k-1}^{-} \subseteq \mathcal{F}_{\bar{x}_{1}}\right|_{X}\right\}$ has size at least $\left(c^{\prime}-\frac{c^{\prime}+1 / 2}{2}\right)\left(\begin{array}{l}n-2 \\ k-2\end{array}\right)=\frac{c^{\prime}-1 / 2}{2}\left(\begin{array}{l}n-2 \\ k-2\end{array}\right)$. If two sets $X_{1}, X_{2} \in \mathcal{G}$ are disjoint, then writing $F_{1}=X_{1} \cup\left\{x_{1}\right\}, F_{2}=X_{2} \cup\left\{x_{1}\right\}$ both $\left.\mathcal{F}\right|_{F_{1}}$ and $\left.\mathcal{F}\right|_{F_{2}}$ contain $\mathcal{C}_{k}^{-}$as $\left.F_{1}\right|_{F_{2}}=F_{1} \cap F_{2}=\left.F_{2}\right|_{F_{1}}=\{x\}$. Thus we may assume that $\mathcal{G}$ is intersecting and thus by Theorem 1.4 and (1) there exists an $x_{2} \in[n] \backslash\left\{x_{1}\right\}$ such that $x_{2} \in X$ for all $X \in \mathcal{G}$.

Let us assume that there is a set $F^{\prime} \in \mathcal{F}_{x_{1}}$ with $x_{2} \notin F^{\prime}$. We claim that there is a set $X \in \mathcal{G}$ such that $F^{\prime} \cap X=\emptyset$. Indeed, the number of $(k-1)$-sets containing $x_{2}$ and meeting $F$ is $\left(\begin{array}{c}n-2 \\ k-2\end{array}\right)-\left(\begin{array}{c}n-k-2 \\ k-2\end{array}\right)$, thus again by (1) there is a set $X \in \mathcal{G}$ as claimed. By the definition of $\mathcal{G}$ there are sets $F_{2}, F_{3}, \ldots, F_{k} \in \mathcal{F}_{x_{1}}$ such that their traces on $X$ form a $\mathcal{C}_{k-1}^{-}$. Writing $F=X \cup\{x\}$ we have $\left.F^{\prime}\right|_{F}=\left\{x_{1}\right\}$ and thus the traces of $F^{\prime}, F_{2}, F_{3}, \ldots, F_{k}$ on $F$ form a $\mathcal{C}_{k}^{-}$proving the lemma in this case.

Otherwise all sets in $\mathcal{F}_{x_{1}}$ contain $x_{2}$ and thus as $x_{1}$ is of maximum degree $x_{1}$ and $x_{2}$ are contained in the same sets of $\mathcal{F}$. The number of sets in $\mathcal{F}$ containing both $x_{1}$ and $x_{2}$ is at most $\left(\begin{array}{l}n-2 \\ k-2\end{array}\right)$, thus removing these sets from $\mathcal{F}$ there remains a family $\mathcal{F}^{1}$ of subsets of $[n] \backslash\left\{x_{1}, x_{2}\right\}$ of size at least

$$
\begin{aligned}
c^{\prime}\left(\begin{array}{l}
n-1 \\
k-1
\end{array}\right) & -\left(\begin{array}{l}
n-2 \\
k-2
\end{array}\right) \\
& =c^{\prime}\left(\left(\begin{array}{l}
n-1 \\
k-1
\end{array}\right)-\left(\begin{array}{l}
n-2 \\
k-1
\end{array}\right)+\left(\begin{array}{l}
n-2 \\
k-1
\end{array}\right)-\left(\begin{array}{l}
n-3 \\
k-1
\end{array}\right)\right)-\left(\begin{array}{l}
n-2 \\
k-2
\end{array}\right)+c^{\prime}\left(\begin{array}{l}
n-3 \\
k-1
\end{array}\right) \\
& =c^{\prime}\left(\left(\begin{array}{l}
n-2 \\
k-2
\end{array}\right)+\left(\begin{array}{l}
n-3 \\
k-2
\end{array}\right)\right)-\left(\begin{array}{l}
n-2 \\
k-2
\end{array}\right)+c^{\prime}\left(\begin{array}{l}
n-3 \\
k-1
\end{array}\right) \geq c^{\prime}\left(\begin{array}{l}
n-3 \\
k-1
\end{array}\right)+1,
\end{aligned}
$$

where the last inequality follows by (2).

Let us consider an element $x_{3} \in[n] \backslash\left\{x_{1}, x_{2}\right\}$ with maximum degree in $\mathcal{F}^{1}$. Repeating the above argument we either find a set $X \subset[n] \backslash\left\{x_{1}, x_{2}\right\}$ such that $\left.\left.\mathcal{C}_{k}^{-} \subset \mathcal{F}_{x_{3}}^{1}\right|_{X} \subset \mathcal{F}\right|_{X}$ or we have an element $x_{4} \in[n] \backslash\left\{x_{1}, x_{2}, x_{3}\right\}$ such that $x_{3}$ and $x_{4}$ are contained in exactly the same sets of $\mathcal{F}^{1}$. Removing these sets from $\mathcal{F}^{1}$ we obtain a family $\mathcal{F}^{2} \subset\left(\begin{array}{c}{[n] \backslash\left\{x_{1}, x_{2}, x_{3}, x_{4}\right\}} \\ k\end{array}\right)$ with size at least $\left|\mathcal{F}^{1}\right|-\left(\begin{array}{l}n-4 \\ k-2\end{array}\right) \geq c^{\prime}\left(\begin{array}{l}n-3 \\ k-1\end{array}\right)-\left(\begin{array}{l}n-4 \\ k-2\end{array}\right)+1$ which is by (2) greater or equal to $c^{\prime}\left(\begin{array}{l}n-5 \\ k-1\end{array}\right)+1+1=c^{\prime}\left(\begin{array}{l}n-5 \\ k-1\end{array}\right)+2$. 
Repeating the above argument $l$ times, we either find a set $X$ such that $\left.\mathcal{C}_{k}^{-} \subseteq \mathcal{F}^{l-1}\right|_{X} \subseteq$ $\left.\mathcal{F}\right|_{X}$ or subfamily $\mathcal{F}^{l} \subseteq\left(\begin{array}{c}{[n] \backslash\left\{x_{1}, x_{2}, z_{3}, x_{4}, \ldots, x_{2 l-1}, x_{2 l}\right\}} \\ k\end{array}\right) \cap \mathcal{F}^{l-1}$ with size at least $c^{\prime}\left(\begin{array}{c}n-2 l-1 \\ k-1\end{array}\right)+l$. Thus we either find a set $X$ such that $\left.\left.\mathcal{C}_{k}^{-} \subseteq \mathcal{F}^{l}\right|_{X} \subseteq \mathcal{F}\right|_{X}$ for some $l \leq \frac{n-M}{2}$ or as $n \geq M+1+2\left(\begin{array}{c}M+1 \\ k-1\end{array}\right)$ we obtain a subfamily of $\mathcal{F}$ on $M$ or $M+1$ elements (depending on the parity of $n$ ) with size larger than $\left(\begin{array}{c}M+1 \\ k-1\end{array}\right)$, and thus by Theorem 1.2 we even find a $2^{[k]}$ as trace which proves the lemma.

To prove the theorem for some $k$ and $c$, let us fix an integer $N(k, c)$ larger than $N^{\prime}\left(k, \frac{c+1 / 2}{2}\right)$ of the Lemma such that for any $n \geq N(k, c)$ the following inequality holds

$$
\frac{c-1 / 2}{2}\left(\begin{array}{l}
n-1 \\
k-1
\end{array}\right)>\left(\begin{array}{l}
n-1 \\
k-1
\end{array}\right)-\left(\begin{array}{c}
n-k-1 \\
k-1
\end{array}\right)+1 .
$$

Let $\mathcal{F} \subset\left(\begin{array}{c}{[n]} \\ k\end{array}\right)$ be a family with size at least $c\left(\begin{array}{c}n-1 \\ k-1\end{array}\right)$. We claim that the size of the set $\mathcal{H}=\left\{X \subset[n]:|X|=k, \mathcal{C}_{k}^{-} \subseteq \mathcal{F}_{X}\right\}$ is at least $\frac{c-1 / 2}{2}\left(\begin{array}{l}n-1 \\ k-1\end{array}\right)$. Indeed, using Lemma 2.1 to $\mathcal{F}$ we obtain 1 set in $\mathcal{H}$, then removing this set from $\mathcal{F}$ and applying the Lemma again we get another set and so on until the remaining family contains less set than $\frac{c+1 / 2}{2}\left(\begin{array}{c}n-1 \\ k-1\end{array}\right)$ sets. If there is a pair of disjoint sets $X_{1}, X_{2} \in \mathcal{H}$, then $X_{1} \cap X_{2}=\emptyset$ extends this to a $\mathcal{C}_{k}$, thus we may assume that those sets form an intersecting family, therefore by Theorem 1.4 and (3) there must exist an element $x \in[n]$ such that $x \in X$ for all $X \in \mathcal{H}$. Any set $F \in \mathcal{F} \backslash \mathcal{H}$ must meet all sets in $\mathcal{H}$ as otherwise $\left.F\right|_{X}=F \cap X=\emptyset$ would complete $\left.\mathcal{C}_{k}^{-} \subseteq \mathcal{F}\right|_{X}$ to $\mathcal{C}_{k}$. But this can happen only if $F$ contains $x$ as otherwise the number of $k$-sets containing $x$ and meeting $F$ would be $\left(\begin{array}{c}n-1 \\ k-1\end{array}\right)-\left(\begin{array}{c}n-k-1 \\ k-1\end{array}\right)$ which is by (3) smaller than $\frac{c-1 / 2}{2}\left(\begin{array}{c}n-1 \\ k-1\end{array}\right) \leq|\mathcal{H}|$. Thus all sets in $\mathcal{F}$ contain $x$ which proves the theorem.

\section{Remark}

Frankl and Watanabe [6] strengthened the conjecture of Frankl and Pach to the following: for every $k \leq m$ there exists an $N=N(k, m)$ such that for any $n \geq N$ and family $\mathcal{F} \subseteq\left(\begin{array}{c}{[n]} \\ m\end{array}\right)$ with size larger than $\left(\begin{array}{c}n-m+k-1 \\ k-1\end{array}\right)$ there is a $k$-subset $X$ of $[n]$ such that $2^{[k]}=\left.\mathcal{F}\right|_{X}$. The counterexample of Ahlswede and Khachatrian can be extended to the $k<m$ case. It is natural to ask what happens if we change again $2^{[k]}$ to $\mathcal{C}_{k}$. Our proof does not carry through mainly because of two reasons: $X_{1} \cap X_{2}=\emptyset,\left|X_{1}\right|=\left|X_{2}\right|=k$, $\mathcal{F} \subseteq\left(\begin{array}{c}{[n]} \\ m\end{array}\right),\left.\mathcal{C}_{k}^{-} \subseteq \mathcal{F}\right|_{X_{1}},\left.\mathcal{F}\right|_{X_{2}}$ does not imply $\left.\mathcal{C}_{k} \subseteq \mathcal{F}\right|_{X_{1}}$ if $k<m$ and two different $m$-sets $F_{1}, F_{2}$ may have $\left.F_{1}\right|_{X}=\left.F_{2}\right|_{X}=X$ for some $k$-set $X$. However, we conjecture that an analogous statement for the $k<m$ case is true.

Conjecture 2.2. For any pair of integers $2 \leq k \leq m$ and $\mathcal{F} \subseteq\left(\begin{array}{c}{[n]} \\ m\end{array}\right)$ with $|\mathcal{F}|>\left(\begin{array}{c}n-m+k-1 \\ k-1\end{array}\right)$ there exists a $k$-subset $X$ of $[n]$ such that $\left.\mathcal{C}_{k} \subseteq \mathcal{F}\right|_{X}$.

The bound (if true) would be sharp as shown by the family $\left\{F \in\left(\begin{array}{c}{[n]} \\ m\end{array}\right):[m-k+1] \subset F\right\}$.

Acknowledgement. The author would like to thank the anonymous referee for his/her careful reading and valuable comments. 


\section{References}

[1] R. Ahlswede, L. Khatchatrian, Counterexample to the Frankl-Pach conjecture for uniform, dense families, Combinatorica 17 (1997), 299-301.

[2] J. Balogh, B. Bollobás, Unavoidable traces of set systems, Combinatorica, 25 (2005), 633-643.

[3] J. Balogh, P. Keevash, B. Sudakov, Disjoint representability of sets and their complements, Journal of Combinatorial Theory B, 95 (2005), 12-28.

[4] P. Erdős, C. Ko, R. RADO, Intersection theorems for systems of finite sets, Quart. J. Math. Oxford, 12 (1961), 313-318.

[5] P. Frankl, J. Pach, On disjointly representable sets. Combinatorica 4 (1984), 39-45.

[6] P. Frankl, M. Watanabe, Density results for uniform families, Combinatorica 14 (1994), 115-119.

[7] Z. Füredi, F. Quinn, Traces of finite sets, Ars Combin., 18 (1984) 195-200.

[8] A.J.W. Hilton, E.C. Milner, Some intersection theorems for systems of finite sets, Quart. J. Math. Oxford, 18 (1967), 369-384.

[9] D. Mubayi, J. ZhaO, On the VC-dimension of uniform hypergraphs, Journal of Algebraic Combinatorics 25 (2007) 101-110.

[10] B. Patkós, $l$-trace $k$-Sperner families, submitted.

[11] N. Sauer, On the density of families of sets, Journal of Combinatorial Theory A 13 (1972), 145-147.

[12] S. Shelah, A combinatorial problem; stability and order for models and theories in infinitary languages, Pacific J. Math 41 (1972), 271-276.

[13] V.N. Vapnik, A. Ya. Chervonenkis, The uniform convergence of relative frequencies of events to their probabilities, Theory Probab Appl. 16 (1971), 264-279. 